\title{
Experience-dependent associations between distinct subtypes of childhood trauma and brain function and architecture
}

\author{
Jia Li ${ }^{1} \wedge$, Guiling Zhang ${ }^{2}$, Jian Wang ${ }^{1}$, Dong Liu ${ }^{1}$, Changhua Wan ${ }^{1}$, Jicheng Fang ${ }^{1}$, Di Wu ${ }^{2}$, Yiran Zhou ${ }^{2}$, \\ Tian $\operatorname{Tian}^{1}$, Wenzhen Zhu ${ }^{1}$ \\ ${ }^{1}$ Department of Radiology, Tongji Hospital, Tongji Medical College, Huazhong University of Science and Technology, Wuhan, China; ${ }^{2}$ Tongji \\ Medical College, Huazhong University of Science and Technology, Wuhan, China
}

Contributions: (I) Conception and design: J Li, T Tian; (II) Administrative support: W Zhu; (III) Provision of study materials or patients: W Zhu; (IV) Collection and assembly of data: G Zhang, J Wang, D Liu, C Wan, J Fang, Y Zhou, D Wu; (V) Data analysis and interpretation: J Li, T Tian; (VI) Manuscript writing: All authors; (VII) Final approval of manuscript: All authors.

Correspondence to: Tian Tian, MD; Wenzhen Zhu, PhD. Department of Radiology, Tongji Hospital, Tongji Medical College, Huazhong University of Science and Technology, 1095 Jiefang Ave, Wuhan 430030, China. Email: tongjitiantian@163.com; zhuwenzhen8612@163.com.

Background: Childhood trauma can alter brain-development trajectories and lead to a greater risk of psychopathology developing in adulthood. For this reason, understanding experience-dependent brain abnormalities associated with different trauma subtypes is crucial for identifying developmental processes disrupted by unfavorable early environments and for proposing early intervention measures to reduce trauma's negative effects.

Methods: This study used multimodal magnetic resonance imaging (MRI) to explore the neural correlates of distinct subtypes of childhood trauma. We recruited a large community sample of young adults (mean age, 24.1, SD 1.9 years) who completed a Childhood Trauma Questionnaire, were given behavioral scores, and underwent multimodal MRI. To quantify brain changes, we used functional connectivity density (FCD) mapping based on whole brain analysis, regions of interest (ROI) analysis, and morphological measurements. Experience-dependent brain abnormalities were identified by multivariable linear regression.

Results: We found that diverse brain regions in the FCD mapping were significantly related to 4 trauma subtypes and belonged to different cognitive components used for various behaviors. Experience-related influences on functional circuits and brain morphology were observed in extensive regions, including the sensorimotor, cingulum, accumbens, insula, and frontal-parietal areas, as well as in regions within the default mode network.

Conclusions: Identifying specific regions or systems may be a valid strategy for understanding the pathogenesis and development process of psychiatric disorders in people with different traumatic experiences and may facilitate better-targeted intervention strategies for maltreated children.

Keywords: Abuse; neglect; functional connectivity; cognition; emotion regulation

Submitted Apr 23, 2021. Accepted for publication Aug 31, 2021.

doi: 10.21037/qims-21-435

View this article at: https://dx.doi.org/10.21037/qims-21-435

\footnotetext{
^ ORCID: 0000-0003-4706-2068.
} 


\section{Introduction}

Childhood trauma, such as emotional abuse or neglect, physical abuse or neglect, and sexual abuse are prevalent in today's world (1). These early trauma experiences can have long-term effects on mental health later in adulthood. Thus far, the neurobiological mechanisms responsible for these long-term effects remain unclear. For this reason, neuroimaging is an ideal, noninvasive way of exploring brain responses to environmental effects in vivo. Many neuroimaging studies have indicated that altered braindevelopment trajectories relate to childhood trauma experiences and may lead to a greater risk for adults to develop mental disorders (2-5). This is due to childhood trauma producing a series of hormonal physiological changes during sensitive or critical periods, leading to excessive overstimulation to neurons and resulting in altered brain structure and function (6). Several vital regions that modulate reward processing, emotional stimulation, cognitive regulation, and executive function are altered in trauma-exposed individuals, particularly in the prefrontal cortex, cingulate gyrus, amygdala, insula, and hippocampus (5,7-12). Specific network architectures or pathways that dominate conscious perception, emotion regulation, threat detection, defense response, and reward anticipation are also affected, including the default mode network (13), emotion circuitry (14), limbic network (11,15), salience network (16), fiber pathway (17-21), and global white matter network (22).

However, distinct dimensions of abuse and neglect experiences may have different neurobiological effects $(23,24)$. In recent years, a growing number of studies have been carried out to systematically examine the effects of 5 types of childhood adversity on brain modulations (20,25-27). An abundance of evidence suggests that different forms of trauma have distinct influences on brain architectures and circuits, as well as on neural development (28-30). Abusive and neglectful forms of childhood trauma differ in their associations with atypical neural functioning (31). For example, threatening contexts increase defense responses and dysregulation of reward processing in regions like the thalamus and striatum, while deprivation disrupts aspects of cognitive and executive function modulated by executive networks (29,32-34). Furthermore, the risk for psychopathology increases in a graded manner as the number of adverse childhood experiences increases (35). On this account, understanding experience-dependent brain abnormalities associated with each trauma subtype is of crucial importance to identifying those developmental processes disrupted by unfavorable early environments and to proposing early interventions the negative effects of trauma.

Moreover, based on a strong link between childhood trauma and the increased risk of psychopathology, many previous studies have explored the associations between early trauma, brain changes, and the onset or severity of psychiatric disorders in patients, such as depressive disorders (5), posttraumatic stress disorder (7), anxiety (10), and borderline personality disorder (36). The most prominent limitation of these studies is that the impact of childhood trauma, psychiatric condition, or their interaction on the brain remains uncertain. The existence and nature of biological alterations due to childhood trauma (beyond its impact on different psychiatric disorders) are even less clear. In contrast, research based on a community sample or healthy groups may have certain advantages in exploring the nature of biological alterations of childhood trauma and the potential trauma-related neural mechanisms that lead to psychiatric disorders (20,37-40).

For this study, we used integrated multimodal of restingstate functional MRI (fMRI) and structural MRI (sMRI) to study the experience-dependent associations between distinct subtypes of childhood trauma, brain function, and architecture in a large community sample of young adults. All participants completed a Childhood Trauma Questionnaire (CTQ), were given behavioral scores, and underwent a multimodal MRI scan. The restingstate functional connectivity strength is one of the most widely used tools to study biological characteristics of brain changes (41). Defined as the statistical dependence of neurophysiological activity between 2 independent brain regions, the level of correlation between different brain areas describes the strength of underlying interregional functional pathways (42). Functional connectivity density (FCD) mapping is a data-driven method that quantifies the number of local and global functional connections for each voxel in the brain (43). The unique effects that CTQ subtypes have on FCD can help identify different cognitive components used for diverse behaviors and thereby yield finer-grained insights into the organization of brain function (44). Another important feature of brain development is the behavioral changes and related pathologies that occur in modifications of brain morphology (45). The various features extracted from functional and morphological analyses can provide objective evidence for the link between childhood trauma subtypes and brain-behavior alterations. We hypothesize that each different subtype of childhood trauma exerts 
dissimilarly profound influences on brain structure and function. Thus, identifying specific regions or systems may be a valid strategy to understanding the pathogenesis and development process of psychiatric disorders in victims with different traumatic experiences. The following article is presented in accordance with the Materials Design Analysis Reporting (MDAR) checklist (available at https://dx.doi. org/10.21037/qims-21-435).

\section{Methods}

\section{Participants}

A total of 216 right-handed, healthy, young adults (aged 20 to 30 years) were recruited for this study. All subjects completed a structured clinical interview as our previous study (46) to ensure that they had no history of psychiatric or neurological illness, psychiatric treatment, or drug or alcohol abuse, and no contraindications for MRI examination. It was approved by the Ethical Committee of Tongji Hospital of Tongji Medical College of Huazhong University of Science and Technology (no. TJ-C20151204). All participants gave written informed consent. The study was conducted in accordance with the Declaration of Helsinki (as revised in 2013). All methods were carried out in accordance with approved institutional guidelines and regulations.

\section{Questionnaires}

Every adult in our study was assessed for experiences of childhood maltreatment through the Chinese version of the short-form CTQ $(47,48)$. To further characterize the participants, the California Verbal Learning TestSecond Edition (CVLT-II), Tridimensional Personality Questionnaire (TPQ), Beck Depression Inventory (BDI), and Spielberger's State-Trait Anxiety Inventory (STAI) were also completed.

\section{MRI scans and data preprocessing procedure}

All scans were performed on a 3.0-Tesla MR system (Discovery MR750, GE Healthcare, Chicago, IL, USA). Tight but comfortable foam padding was used to minimize head motion, and earplugs were used to reduce scanner noise. Resting-state fMRI data were obtained using singleshot echo-planar imaging (SS-EPI), and sagittal 3D T1weighted images were acquired using a brain volume (BRAVO) sequence. The detailed MRI scan parameters and data preprocessing procedure were the same as those used in our previous study (46).

\section{FCD calculation}

Compared with hypothesis-driven resting-state functional connectivity analysis, voxel-wise FCD mapping does not need to assess relationships between brain regions or select seed points. This data-driven graph theory approach quantifies global and local spontaneous neuronal activity and connectivity of voxels over the whole brain, which leads to faster calculation speeds, more accurate results, and provides complementary information for more targeted analysis (49). Generally, a higher FCD value indicates that there are more functional connections between related areas and other areas (50). It is also regarded as a functional property in hub recognition. We calculated the FCD of each voxel using the in-house script of the Linux platform according to the method described by Tomasi and Volkow (43). The detailed FCD calculation procedure was the same as that in our previous study (51).

\section{Regions of interest definition and functional connectivity analysis}

After preprocessing, fMRI data were smoothed using a fullwidth Gaussian kernel of $8 \times 8 \times 8 \mathrm{~mm}^{3}$ at half-maximum. All 91 cortical regions from the FMRIB Software Library (FSL) Harvard-Oxford Atlas maximum likelihood cortical atlas (HarvardOxford-cort-maxprob-thr25-1mm.nii) and 15 subcortical regions from the FSL Harvard-Oxford Atlas maximum likelihood subcortical atlas (HarvardOxford-submaxprob-thr25-1mm.nii) were defined as regions of interest (ROIs). We calculated the functional connectivity strength between each pair in the 106 ROIs and obtained a 106×106 matrix for each subject. The functional connectivity strength was computed using Pearson correlation coefficient to separately determine the average time series between the 2 ROIs. The correlation coefficient was then transformed to approximate a Gaussian distribution using Fisher's r-to-z transformation.

Atlases of 7 commonly used cortical networks, including the default mode network, sensorimotor network, visual network, salience network, dorsal attention network, frontoparietal network, and language network, were defined from CONN's independent component analyses of the Human Connectome Project data set (52). We computed the functional connectivity between each pair of the 7 
network masks in the same way as above. Finally, for each participant, we obtained a $7 \times 7$ matrix, with each element representing the mean connectivity strength between the corresponding 2 networks.

\section{Morphological measurements}

The cortical parcellation was created in FreeSurfer (version 6.0.0) software's standard anatomical template (fsaverage) space using the Desikan-Killiany-Tourville (DKT) atlas, with 31 cortical regions per hemisphere (53) subsequently transformed to each individual participant's surface. For each region, we estimated grey matter volume, surface area, and cortical thickness.

\section{Statistical analysis}

We assessed the unique effects of the different CTQ subtypes on FCD mapping using a multivariable linear regression model (SPM8 software). Education level, age, and sex were applied in our statistical model as covariates (54). Cluster-level family-wise error (FWE) correction $\left(\mathrm{P}_{\text {adj }}<0.05\right)$ was used to correct multiple comparisons. Surviving clusters were saved as masks. Unique effects of different CTQ subtypes on surviving clusters were adopted for the subsequent identification of the different cognitive components which are used for diverse behaviors. For each specific cognitive component obtained from Yeo et al. (44), we projected each significant cluster onto the cognitive map to determine whether the cluster was located in the cognitive component.

Data obtained from the connectivity strength analysis then underwent a Kolmogorov-Smirnov test for data distribution. A linear regression model was used to assess the relationships between the functional connectivity of ROIs/cortical networks and all CTQ subtypes, while gender, education level, and age were adopted for the multivariable regression analyses. Because 5 hypotheses were tested simultaneously and a null hypothesis could have been wrongly rejected by chance, we used the BenjaminiHochberg procedure to control the false discovery rate (FDR) in the multiple-CTQ subtype models and applied a significant threshold of 2 -sided $\mathrm{P}_{\text {adj }}$ value $<0.05$. All analyses were performed using $\mathrm{R}$ (version 3.6.2, The R Foundation for Statistical Computing).

Data obtained from the sMRI also underwent a Kolmogorov-Smirnov test for data distribution. We used multivariable linear regression analysis to evaluate the associations between the anatomical features of 62 cortical regions and the different CTQ subtypes, with adjustments for gender, education level, age, and total intracranial volume (54). All analyses were performed using R (version 3.6.2). Similarly, FDR correction $\left(\mathrm{P}_{\text {adj }}<0.05\right)$ was used for multiple comparisons.

To test a single risk, we used partial correlation analysis to study correlations between behavioral scores and each CTQ subtype using SPSS (version 19.0, IBM Corporation, Armonk, NY, USA), while the other 4 subscales, gender, education level, and age, were controlled for in the models.

\section{Results}

\section{Demographic and behavioral characteristics}

Demographic and behavioral characteristics of the sample $(n=216)$ can be seen in Table 1. Correlation analyses showed that the emotional abuse score was positively correlated with the state anxiety score $(\mathrm{P}=0.006, \mathrm{r}=0.189)$, trait anxiety score $(\mathrm{P}=0.008, \mathrm{r}=0.184)$, and $\mathrm{BDI}$ score $(\mathrm{P}=0.026, \mathrm{r}=0.154)$, while the emotional neglect score was positively correlated with the harm avoidance score $(\mathrm{P}=0.009, \mathrm{r}=0.180)$. There were no significant correlations between the CTQ subtypes and verbal memory $(\mathrm{P}>0.05)$.

\section{Unique effects of different CTQ subtypes on FCD mapping and related cognitive components}

Significant associations of emotional abuse (Figure 1A), physical abuse (Figure 1B), sexual abuse (Figure 1C), and physical neglect (Figure 1D) with different regions were found in FCD mapping. Related cognitive components of these regions are also shown in Figure 1. The detailed statistical information is shown in Table 2. No significant effect of emotional neglect on FCD mapping was observed.

\section{Experience-dependent associations between distinct subtypes of childbood trauma and functional connectivity}

A linear regression model using functional connectivity between ROIs as dependent variables and all CTQ subtypes as independent variables, defined the final model. The distribution of the 106 ROIs is displayed in Figure $2 \mathrm{~A}$. Significant associations of 4 CTQ subtypes with functional connectivity between ROIs are provided in Figure $2 B$. More detailed statistical information for the significant associations of CTQ subtypes with functional connectivity between ROIs is shown in Table 3. 
Table 1 Demographic and behavioral characteristics of the sample group

\begin{tabular}{|c|c|c|}
\hline Demographics & Mean (SD) & Range \\
\hline Age (years) & $24.1(1.9)$ & $20-30$ \\
\hline Gender (female/male) & $158 / 58$ & - \\
\hline Education level (years) & $17.7(1.5)$ & $13-22$ \\
\hline CTQ sum score & $30.4(5.2)$ & $25-49$ \\
\hline Emotional abuse & $6.2(1.5)$ & $5-11$ \\
\hline Physical abuse & $5.5(1.3)$ & $5-14$ \\
\hline Sexual abuse & $5.2(0.6)$ & $5-9$ \\
\hline Emotional neglect & $7.7(2.7)$ & $5-17$ \\
\hline Physical neglect & $5.9(1.4)$ & $5-11$ \\
\hline BDI score & $4.7(4.9)$ & $0-22$ \\
\hline STAI score & $69.1(14.8)$ & $42-119$ \\
\hline Novelty seeking & $13.9(4.4)$ & $4-29$ \\
\hline Harm avoidance & $15.2(5.6)$ & $2-30$ \\
\hline Reward dependence & $19.1(3.4)$ & $10-27$ \\
\hline \multicolumn{3}{|l|}{ Verbal memory } \\
\hline Learning & $59.4(8.6)$ & $23-79$ \\
\hline Recall & $13.4(2.2)$ & $5-16$ \\
\hline Recognition & $15.5(0.9)$ & $11-16$ \\
\hline List $A-1$ & $7.6(2.0)$ & $3-15$ \\
\hline List B & $7.3(2.3)$ & $3-14$ \\
\hline False-positive errors & $1.1(1.9)$ & $0-10$ \\
\hline
\end{tabular}

BDI, Beck Depression Inventory; CTQ, Childhood Trauma Questionnaire; SD, standard deviation; STAI, State-Trait Anxiety Inventory.

Similarly, a linear regression model was performed to explore the associations of functional connectivity between 7 classical networks with CTQ subtypes. There were no significant results after multiple comparisons correction $\left(\mathrm{P}_{\mathrm{adj}}<0.05\right)$.

\section{Experience-dependent associations between distinct subtypes of childhood trauma and brain morphology}

A linear regression model using morphological features of 62 cortical regions as dependent variables and all CTQ subtypes as independent variables defined the final model. After FDR correction $\left(\mathrm{P}_{\mathrm{adj}}<0.05\right)$ of the multiple comparisons, the significant associations of 5 CTQ subtypes with cortical regions were obtained. These data are demonstrated with different colors in Figure 3. More detailed statistical information is shown in Table 4.

\section{Discussion}

We investigated diverse effects of different subtypes of childhood trauma on FCD mapping, related cognitive components, functional circuits, and cortical morphology. We found that diverse brain regions in the FCD mapping were significantly related to 4 trauma subtypes and belonged to different cognitive components used for various behaviors. Experience-related influences on functional circuits and brain morphology were observed in extensive regions, including the sensorimotor, cingulum, accumbens, insula, and frontal-parietal areas, as well as regions within the default mode network. Moreover, we found that the emotional abuse score had a significant positive correlation with anxiety and depression scores, while the emotional neglect score had a significant positive correlation with the harm avoidance score. This study identifies unique effects of subtypes on specific regions or systems and may be a valid strategy to understanding the pathogenesis and development process of psychiatric disorders related to specific subtypes of childhood trauma.

\section{Associations of emotional abuse with changes of regions involved in sensorimotor processing and emotion regulation}

Emotional abuse refers to the rejection of a child's positive emotions by guardians. Emotionally abused children are frequently exposed to verbal assaults, threats, terrorization, or close confinement. We observed that emotional abuse was associated with increased FCD in the postcentral gyrus, which belongs to cognitive components involved in perception, vision, and action. Additionally, the emotional abuse scores correlated with morphological alterations of the orbitofrontal cortex. In humans, a larger morphology of the medial orbitofrontal cortex is positively associated with fear extinction and resiliency (55). Meanwhile, the reduction of pars orbitalis may release select inhibitory control mechanisms and allow for enhanced processing of depressive thoughts or emotions associated with traumatic memories (56). The functional and morphological alterations support potentially abnormal sensorimotor function and emotion regulation in individuals with a history of emotional abuse. 
A

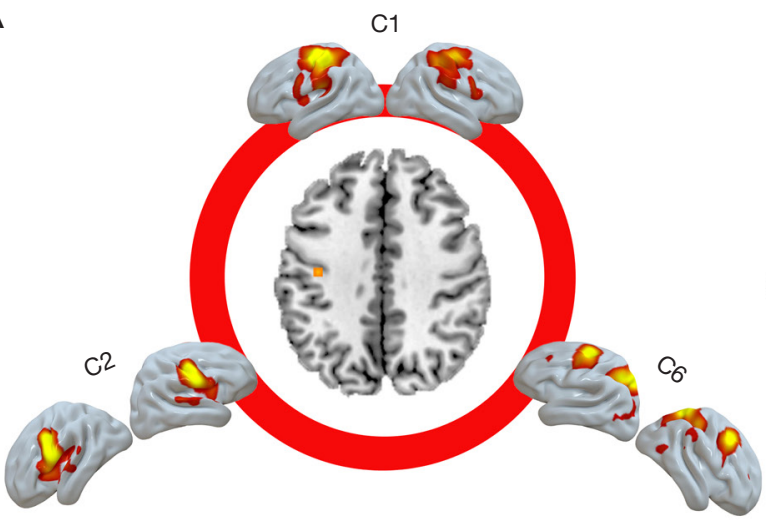

B

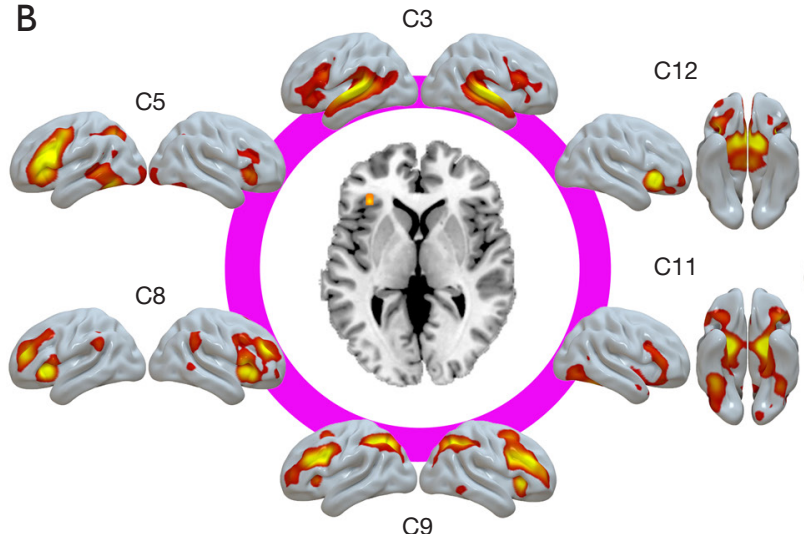

C

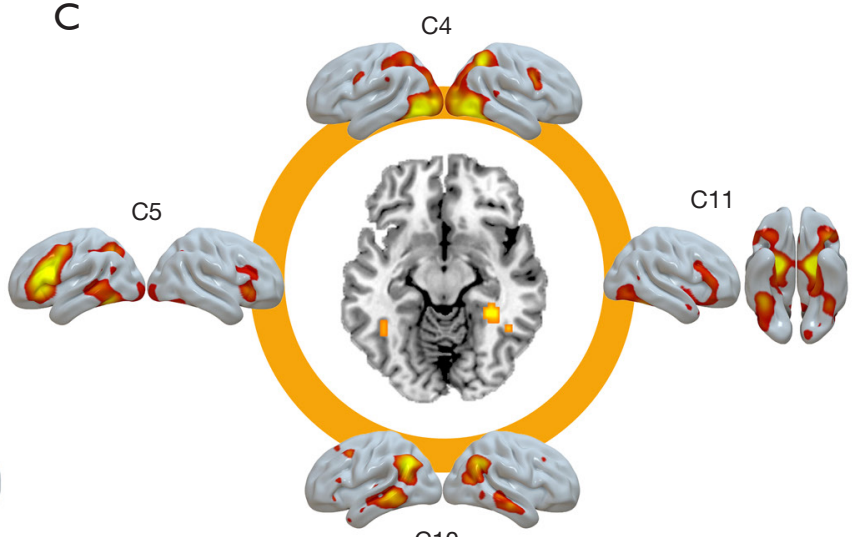

C10

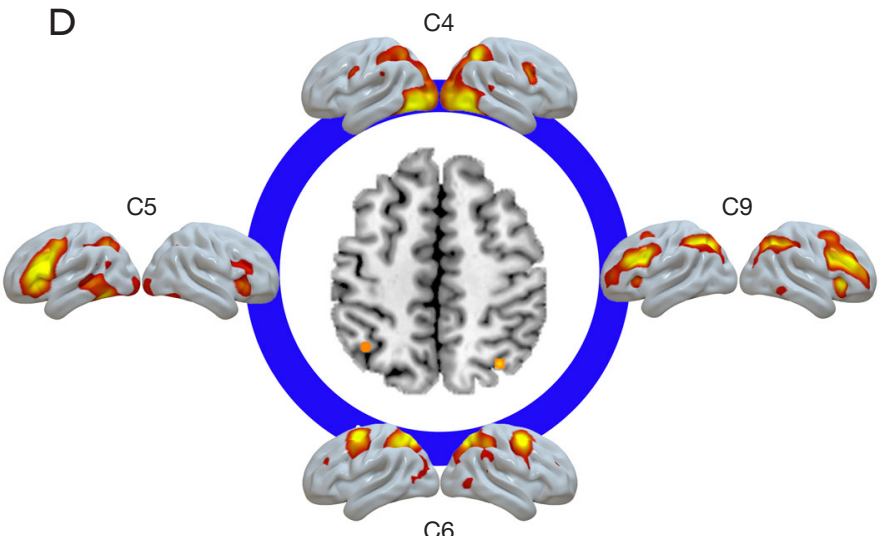

Figure 1 Unique effects of different CTQ subtypes on FCD mapping and related cognitive components. Emotional abuse (A), physical abuse (B), sexual abuse (C), and physical neglect (D) each showed significant associations with different regions in FCD mapping, all of which belong to special cognitive components. CTQ, Childhood Trauma Questionnaire; FCD, functional connectivity density.

Table 2 Significant associations of CTQ subtypes with regions in FCD mapping that belong to specific cognitive components

\begin{tabular}{lllll}
\hline CTQ subtypes & Significant regions in FCD mapping & Peak MNI coordinates $(x, y, z)$ & T value & Related cognitive component \\
\hline Emotional abuse & Left postcentral gyrus & $-39,-15,39$ & 4.96 & $1,2,6$ \\
Physical abuse & Left prefrontal cortex & $-36,33,0$ & 4.92 & $3,5,8,9,11,12$ \\
Sexual abuse & Left temporal lobe & $-42,-51,-6$ & 5.33 & $4,5,10,11$ \\
& Right temporal lobe & $48,-54,-15$ & 5.40 & $4,5,10,11$ \\
& Right parahippocampus & $33,-39,-9$ & 5.83 & $4,5,10,11$ \\
Physical neglect & Left superior parietal lobule & $-24,-72,60$ & 4.95 & $4,5,6,9$ \\
& Right superior parietal lobule & $33,-63,45$ & 5.04 & $4,5,6,9$ \\
\hline
\end{tabular}

CTQ, Childhood Trauma Questionnaire; FCD, functional connectivity density; MNI, Montreal Neurological Institute; Cognitive components are defined by Yeo et al. (44). 
A
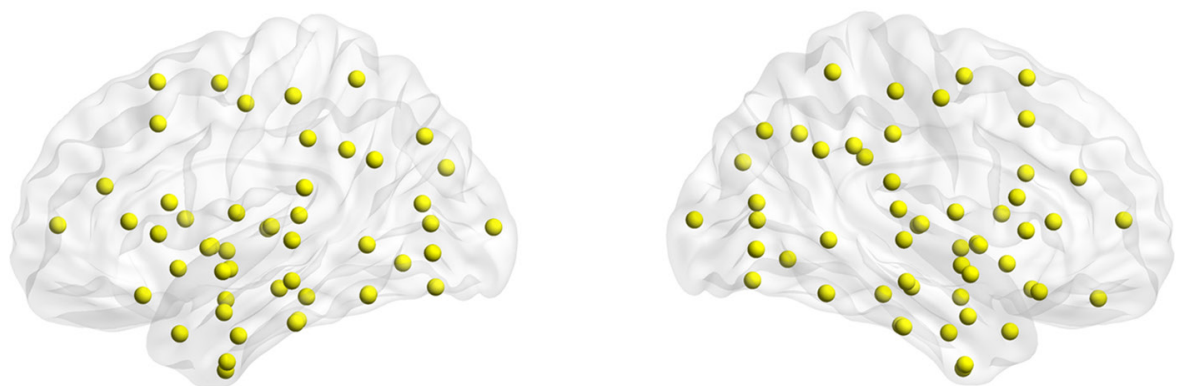

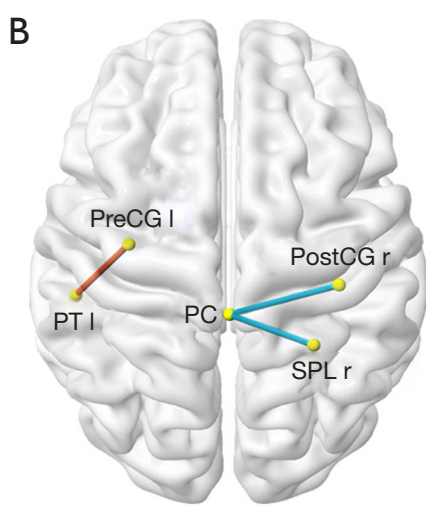

Physical abuse

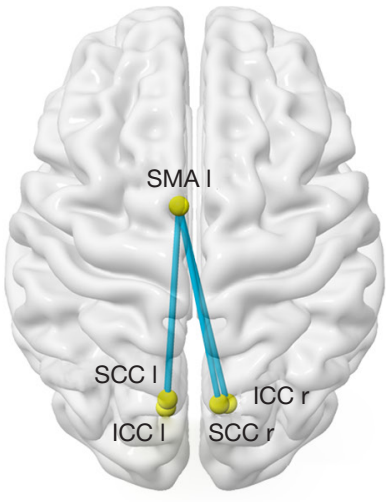

Sexual abuse

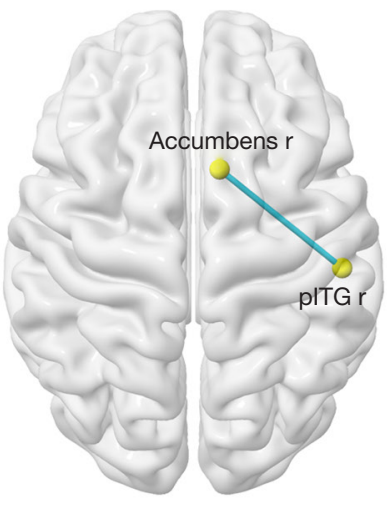

Physical neglect

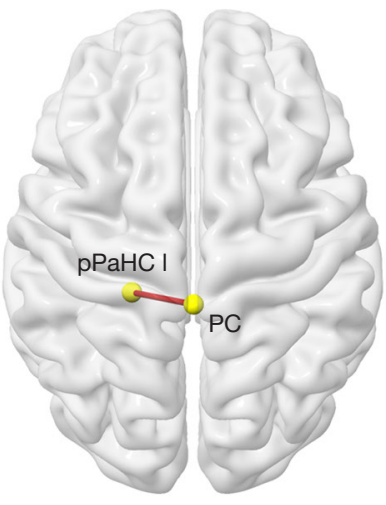

Emotional neglect

Figure 2 Correlations between CTQ subtypes and ROIs connectivity strength. Distribution of 106 ROIs is observed in part A. Significant associations of 4 CTQ subtypes with functional connectivity between ROIs are provided in part B. CTQ, Childhood Trauma Questionnaire; ROI, regions of interest; PostCG r, right postcentral gyrus; PC, posterior cingulate gyrus; SPL r, right superior parietal lobule; PT 1, left planum temporale; PreCG 1, left precentral gyrus; SMA 1, left supplementary motor cortex; SCC 1, left supracalcarine cortex; SCC r, right supracalcarine cortex; ICC 1, left intracalcarine cortex; ICC r, right intracalcarine cortex; pITG r, right posterior division of inferior temporal gyrus; pPaHC 1, left posterior division of parahippocampal gyrus.

Table 3 Significant associations of CTQ subtypes with functional connectivity between ROIs

\begin{tabular}{llll}
\hline CTQ subtypes & ROI-ROI connectivity & Beta $(95 \%$ Cl) & $P_{\text {adjusted value }}$ \\
\hline Physical abuse & PostCG r-PC & $-0.047(-0.070,-0.024)$ & $<0.001$ \\
& SPL r-PC & $-0.048(-0.073,-0.023)$ & 0.002 \\
& PT I-PreCG I & $0.042(0.017,0.066)$ & 0.007 \\
Sexual abuse & SCC I-SMA I & $-0.088(-0.135,-0.041)$ & 0.002 \\
& ICC I-SMA I & $-0.077(-0.123,-0.031)$ & 0.009 \\
ICC r-SMA I & $-0.075(-0.126,-0.024)$ & 0.021 \\
Physical neglect & SCC r-SMA I & $-0.076(-0.126,-0.025)$ & 0.029 \\
Emotional neglect & pITG r-Accumbens r & $-0.033(-0.054,-0.013)$ & 0.005 \\
\hline
\end{tabular}

CTQ, Childhood Trauma Questionnaire; ROI, regions of interest; Cl, confidence interval; PostCG r, right postcentral gyrus; PC, posterior cingulate gyrus; SPL r, right superior parietal lobule; PT I, left planum temporale; PreCG I, left precentral gyrus; SMA I, left supplementary motor cortex; SCC I, left supracalcarine cortex; SCC r, right supracalcarine cortex; ICC I, left intracalcarine cortex; ICC r, right intracalcarine cortex; pITG r, right posterior division of inferior temporal gyrus; $\mathrm{pPaHC}$ I, left posterior division of parahippocampal gyrus. 


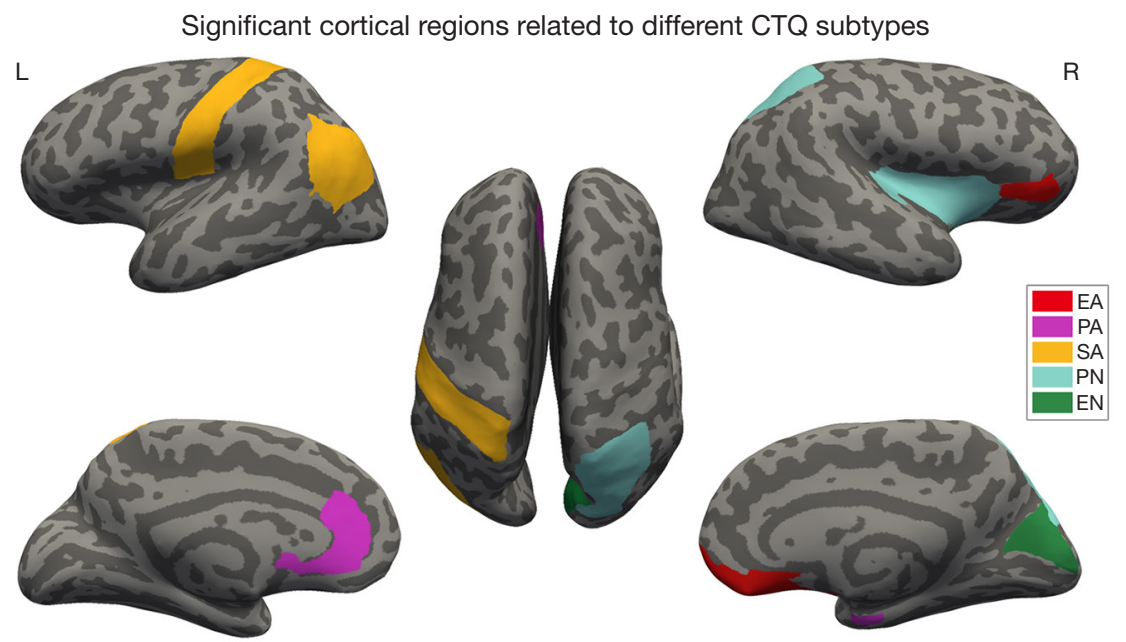

Figure 3 Significant cortical regions related to different CTQ subtypes. Different colored areas of the brain surface represent diverse cortical regions, whose morphological indices are significantly related to the corresponding CTQ subtypes. CTQ, Childhood Trauma Questionnaire; EA, emotional abuse (red); PA, physical abuse (purple); SA, sexual abuse (yellow); PN, physical neglect (blue); EN, emotional neglect (green).

Table 4 Significant associations of CTQ subtypes with cortical morphology

\begin{tabular}{llll}
\hline CTQ subtypes & Cortical regions (indices) & Beta (95\% Cl) & $P_{\text {adjusted value }}$ \\
\hline Emotional abuse & Right medial orbitofrontal cortex (SurfArea) & $25.094(8.049,42.139)$ & 0.011 \\
& Right medial orbitofrontal cortex (GrayVol) & $74.369(23.093,125.644)$ & 0.013 \\
& Right pars orbitalis cortex (ThickAvg) & $-0.024(-0.041,-0.006)$ & 0.032 \\
Physical abuse & Left rostral anterior cingulate cortex (GrayVol) & $75.375(16.092,134.659)$ & 0.029 \\
& Right entorhinal cortex (SurfArea) & $-11.295(-20.261,-2.328)$ & 0.041 \\
Sexual abuse & Left postcentral gyrus (GrayVol) & $-395.693(-661.527,-129.860)$ & 0.011 \\
& Left inferior parietal lobule (ThickAvg) & $-0.031(-0.054,-0.008)$ & 0.033 \\
Physical neglect & Right transverse temporal gyrus (ThickAvg) & $-0.039(-0.062,-0.016)$ & 0.003 \\
& Right superior parietal lobule (GrayVol) & $-180.082(-315.417,-44.748)$ & 0.027 \\
& Right insula cortex (GrayVol) & $-90.885(-162.771,-19.000)$ & 0.040 \\
& Right insula cortex (SurfArea) & $-27.380(-49.142,-5.617)$ & 0.041 \\
Emotional neglect & Right cuneus cortex (SurfArea) & $-20.207(-35.847,-4.568)$ & 0.034 \\
\hline
\end{tabular}

CTQ, Childhood Trauma Questionnaire; Cl, confidence interval; SurfArea, surface area; GrayVol, gray matter volume; ThickAvg, average cortical thickness.

\section{Associations of physical abuse with numerous brain abnormalities}

Physical abuse mainly refers to the physical injury of children caused by inadequate care. Physical abuse was positively correlated with FCD changes in the prefrontal cortex, which belongs to cognitive components mainly involved in auditory processing, language cognition, attention control, affect regulation, face recognition, and reward dependence. First, we found the connectivity between the precentral gyrus and planum temporale to be positively correlated with physical abuse. The precentral 
gyrus is a part of the sensorimotor network, while the planum temporale is thought to be involved in auditory processing, phonological processing, and language (57). Increased connections of the sensorimotor areas reflect the increased sensitivity of sensory discrimination and avoidance strategies to noxious stimuli, known as a part of ego defense mechanisms. Second, we found reduced connectivity in the circuit of the postcentral gyrus, posterior cingulate gyrus, and superior parietal lobule to also be related to physical abuse. The disconnection between these regions in the sensorimotor system and default mode network was reported to contribute to the symptoms of affective disorders in previous studies (58-60).

We also found physical abuse was positively correlated with grey matter volume in the rostral anterior cingulate cortex and was negatively correlated with the surface area of the entorhinal cortex. The changes in the anterior cingulate cortex are related functions in cognitive attention response and pain modulation (61). The entorhinal cortex is thought to contribute to memory retrieval (62). Changes in these regions reveal the reorganization of cognitive reaction, nociceptive processing, and memory coding in response to somatic abusive experiences.

Numerous brain alterations involved in advanced cognitive functions, perception, emotional processing, memory coding, and ego defense mechanisms were found to be associated with physical abuse. This may help us to understand the unique psychopathological consequences related to the experience of physical abuse.

\section{Associations of sexual abuse with disturbances in regions of attention control and sensorimotor processing}

Sexual abuse primarily refers to any sexual exploitation or sexually related assault made directly or indirectly against a child. Our findings showed there to be a positive relationship between sexual abuse and the FCD in the bilateral temporal lobe, which belongs to cognitive components mainly involved in visual tracking, language cognition, and face recognition. Additionally, the sexual abuse subscale was found to be negatively correlated to the functional connectivity between the supplementary motor cortex and calcarine cortex. As an important key of the motor system, the supplementary motor cortex rapidly evaluates successful and erroneous actions, and plays a leading role in the error-monitoring system (63). The calcarine cortex is the focus of the visual cortex and is involved in visual processing (64). The association of sexual abuse with sensorimotor areas in our study supports previous neuroimaging evidence that suggests sexual abuse is associated with abnormalities in sensory processing of maltreatment-specific stimuli.

Sexual abuse was negatively related to the average thickness of the inferior parietal cortex and the grey matter volume of the postcentral gyrus. The structural impairment of the inferior parietal cortex that mediates attention may decrease the ability to switch behavior according to environmental demands (65). The postcentral gyrus is devoted to sensory functions and has been significantly associated with abuse severity (66). The morphological alterations provide the association of sexual abuse with disturbances in attention control and somatic sensation.

\section{Associations of physical neglect with brain abnormalities related to widespread functions}

Physical neglect is defined as the failure of the guardian to provide basic needs for a child to grow up. Increased FCD in the bilateral superior parietal lobule, which belongs to the cognitive components involved in perception, vision, motion, language, execution, and attention, was related to physical neglect. We also found that the physical neglect score was associated with decreased functional connectivity between the accumbens and inferior temporal gyrus. As a functionally central structure, the nucleus accumbens appears to have a modulating effect on the amygdala-basal ganglia-prefrontal cortex circuit and also plays a key role in reward-motivated behavior, depression-related behavior, and substance dependence (67-69). As a part of the visual system, inferior temporal gyrus activation has also been linked to reward processing (70). Reduced functional connectivity between these regions shows the association of physical neglect with the disruption of reward. Furthermore, we found that physical neglect is negatively related to the morphology indices of the transverse temporal cortex, superior parietal lobule, and insula. These regions play important roles in sensory, affective, and attention reactions, as well as in cognitive processing (71). Therefore, we found that a failure to meet the physical needs of children could affect brain areas mainly involved in attention, reward, and sensorimotor processing, as well as affective and social cognitive abilities.

\section{Associations of emotional neglect with altered functions of the default mode network and visual-spatial processing}

Emotional neglect is the failure of guardians to respond to 
the fundamental emotional needs of a child. We observed that the emotional neglect score was associated with an increased connectivity strength in the parahippocampus and posterior cingulate gyrus. The parahippocampus is an advanced center of memory, cognition, and emotion (72), and participates in the default mode network. The posterior cingulate gyrus is a hub of the default mode network and is preferentially vulnerable to disruption in cognitiverelated diseases (73). For individuals who have experienced emotional neglect, increased connectivity between the parahippocampus and posterior cingulate gyrus may be responsible for the atypical functions of the default mode network, including autobiographical memory retrieval, mentalizing, introspective processing, and others (74). Furthermore, we observed that the emotional neglect score was associated with a decreased surface area of the cuneus, which participates in visual-spatial processing (75). Our findings not only support the influences of emotional neglect on memory in the previous study (76) but also further support the associations of emotional neglect with altered functions of the default mode network and visualspatial processing.

\section{Strengths and weaknesses of the study}

Our results revealed that different subtypes of childhood trauma have diverse effects on brain function and architecture, and there are several interesting elements among our findings. First, as an important early environmental factor, different forms of early traumatic experiences exert varying degrees and different expressions of conditioned response to physical and psychological stimuli. To some extent, our results are a record of what the diverse experiences in early life have differently wrought on the brain. Regardless of the increase or decrease in functional connectivity and morphological indexes, typical functions of the brain's regions could be affected to a certain extent. Next, physical abuse and neglect are the subtypes that most affect numerous brain areas related to widespread functions. This suggests that physical trauma has a more comprehensive influence on the brain compared with other forms of trauma. Additionally, there were some overlaps in the different regions and related functions affected by different subtypes. This supports the idea that the degree of brain abnormality increases as the types of adverse childhood experiences increase. In summary, our study provides objective MRI evidence that different forms of early traumatic experiences may differentially and indirectly influence the development of psychopathology by altering the brain. From this, we found that imaging biomarkers may be used to evaluate the brain and develop better-targeted intervention strategies for mediating the negative outcomes experienced by people with different traumatic experiences.

There were several limitations in the current study. First, the study is limited by a retrospective self-report questionnaire of childhood trauma. Although the accuracy may be biased by the subjective experience of the participant, the CTQ has been demonstrated to be a repeatable and valid measure of childhood maltreatment. Second, despite the relatively large community-based sample size, the variation in the levels of childhood maltreatment is limited. Thus, limited significant effects of CTQ subtypes on behavioral scores and network analysis were found. In addition, due to the cross-sectional design of this study, a causal relationship between childhood trauma and brain changes could not be established. For this reason, future longitudinal studies are necessary. Finally, we did not assess the age of subjects at which the trauma occurred or their mental resilience. It will be important for future research to examine if the duration of childhood trauma impacts the risk for psychopathology in adulthood. Furthermore, resilience following childhood maltreatment should be measured to assess protective factors against adult psychopathology.

\section{Conclusions}

This study used a large community sample to examine the experience-dependent associations between distinct subtypes of childhood trauma and brain function and architecture. We found that different forms of early traumatic experiences could exert dissimilarly profound influences on the brain due to the varying degrees and different expressions of conditioned responses to physical and psychological stimuli. For this reason, identifying specific regions or systems may be a valid strategy to understanding the pathogenesis and development process of psychiatric disorders in people with different traumatic experiences. In addition, imaging phenotypes may be useful biomarkers for providing an early assessment of potentially differential risks in adulthood and may guide targeted intervention strategies for avoiding or decreasing the risk of developing psychopathology.

\section{Acknowledgments}

Funding: This study was supported by the National 
Natural Science Foundation of China (No. 81601475 and 81730049).

\section{Footnote}

Reporting Checklist: The authors have completed the MDAR checklist. Available at https://dx.doi.org/10.21037/ qims-21-435

Conflicts of Interest: All authors have completed the ICMJE uniform disclosure form (available at https://dx.doi. org/10.21037/qims-21-435). The authors have no conflicts of interest to declare.

Ethical Statement: The authors are accountable for all aspects of the work in ensuring that questions related to the accuracy or integrity of any part of the work are appropriately investigated and resolved. The study was approved by the Ethical Committee of Tongji Hospital of Tongji Medical College of Huazhong University of Science and Technology (No. TJ-C20151204). All participants gave written informed consent. The study was conducted in accordance with the Declaration of Helsinki (as revised in 2013). All methods were carried out in accordance with approved institutional guidelines and regulations.

Open Access Statement: This is an Open Access article distributed in accordance with the Creative Commons Attribution-NonCommercial-NoDerivs 4.0 International License (CC BY-NC-ND 4.0), which permits the noncommercial replication and distribution of the article with the strict proviso that no changes or edits are made and the original work is properly cited (including links to both the formal publication through the relevant DOI and the license). See: https://creativecommons.org/licenses/by-nc-nd/4.0/.

\section{References}

1. McKay MT, Cannon M, Chambers D, Conroy RM, Coughlan H, Dodd P, Healy C, O'Donnell L, Clarke MC. Childhood trauma and adult mental disorder: A systematic review and meta-analysis of longitudinal cohort studies. Acta Psychiatr Scand 2021;143:189-205.

2. Hart H, Rubia K. Neuroimaging of child abuse: a critical review. Front Hum Neurosci 2012;6:52.

3. Teicher MH, Samson JA, Anderson CM, Ohashi $\mathrm{K}$. The effects of childhood maltreatment on brain structure, function and connectivity. Nat Rev Neurosci
2016;17:652-66.

4. Rakesh D, Kelly C, Vijayakumar N, Zalesky A, Allen NB, Whittle S. Unraveling the Consequences of Childhood Maltreatment: Deviations From Typical Functional Neurodevelopment Mediate the Relationship Between Maltreatment History and Depressive Symptoms. Biol Psychiatry Cogn Neurosci Neuroimaging 2021;6:329-42.

5. Opel N, Redlich R, Dohm K, Zaremba D, Goltermann $\mathrm{J}$, Repple J, et al. Mediation of the influence of childhood maltreatment on depression relapse by cortical structure: a 2-year longitudinal observational study. Lancet Psychiatry 2019;6:318-26.

6. Teicher MH. Scars that won't heal: the neurobiology of child abuse. Sci Am 2002;286:68-75.

7. Klaming R, Spadoni AD, Veltman DJ, Simmons AN. Expansion of hippocampal and amygdala shape in posttraumatic stress and early life stress. Neuroimage Clin 2019;24:101982.

8. Opel N, Redlich R, Repple J, Kaehler C, Grotegerd $\mathrm{D}$, Dohm K, et al. Childhood maltreatment moderates the influence of genetic load for obesity on reward related brain structure and function in major depression. Psychoneuroendocrinology 2019;100:18-26.

9. Cancel A, Dallel S, Zine A, El-Hage W, Fakra E. Understanding the link between childhood trauma and schizophrenia: A systematic review of neuroimaging studies. Neurosci Biobehav Rev 2019;107:492-504.

10. van Velzen LS, Schmaal L, Jansen R, Milaneschi Y, Opmeer EM, Elzinga BM, van der Wee NJ, Veltman DJ, Penninx BW. Effect of childhood maltreatment and brainderived neurotrophic factor on brain morphology. Soc Cogn Affect Neurosci 2016;11:1841-52.

11. Souza-Queiroz J, Boisgontier J, Etain B, Poupon C, Duclap D, d'Albis MA, Daban C, Hamdani N, Le Corvoisier P, Delavest M, Bellivier F, Guevara P, Leboyer M, Henry C, Houenou J. Childhood trauma and the limbic network: a multimodal MRI study in patients with bipolar disorder and controls. J Affect Disord 2016;200:159-64.

12. Johnstone B, Velakoulis D, Yuan CY, Ang A, Steward C, Desmond P, O'Brien TJ. Early childhood trauma and hippocampal volumes in patients with epileptic and psychogenic seizures. Epilepsy Behav 2016;64:180-5.

13. Bluhm RL, Williamson PC, Osuch EA, Frewen PA, Stevens TK, Boksman K, Neufeld RW, Théberge J, Lanius RA. Alterations in default network connectivity in posttraumatic stress disorder related to early-life trauma. J Psychiatry Neurosci 2009;34:187-94.

14. Cisler JM, Privratsky A, Smitherman S, Herringa RJ, Kilts 
CD. Large-scale brain organization during facial emotion processing as a function of early life trauma among adolescent girls. Neuroimage Clin 2017;17:778-85.

15. Cisler JM. Childhood Trauma and Functional Connectivity between Amygdala and Medial Prefrontal Cortex: A Dynamic Functional Connectivity and Large-Scale Network Perspective. Front Syst Neurosci 2017;11:29.

16. van der Werff SJ, Pannekoek JN, Veer IM, van Tol MJ, Aleman A, Veltman DJ, Zitman FG, Rombouts SA, Elzinga BM, van der Wee NJ. Resilience to childhood maltreatment is associated with increased resting-state functional connectivity of the salience network with the lingual gyrus. Child Abuse Negl 2013;37:1021-9.

17. Choi J, Jeong B, Polcari A, Rohan ML, Teicher MH. Reduced fractional anisotropy in the visual limbic pathway of young adults witnessing domestic violence in childhood. Neuroimage 2012;59:1071-9.

18. Jackowski AP, Douglas-Palumberi H, Jackowski M, Win L, Schultz RT, Staib LW, Krystal JH, Kaufman J. Corpus callosum in maltreated children with posttraumatic stress disorder: a diffusion tensor imaging study. Psychiatry Res 2008;162:256-61.

19. Eluvathingal TJ, Chugani HT, Behen ME, Juhász C, Muzik O, Maqbool M, Chugani DC, Makki M. Abnormal brain connectivity in children after early severe socioemotional deprivation: a diffusion tensor imaging study. Pediatrics 2006;117:2093-100.

20. Tendolkar I, Mårtensson J, Kühn S, Klumpers F, Fernández G. Physical neglect during childhood alters white matter connectivity in healthy young males. Hum Brain Mapp 2018;39:1283-90.

21. Monteleone AM, Monteleone P, Esposito F, Prinster A, Ruzzi V, Canna A, Aiello M, Di Salle F, Maj M. The effects of childhood maltreatment on brain structure in adults with eating disorders. World J Biol Psychiatry 2019;20:301-9.

22. Puetz VB, Parker D, Kohn N, Dahmen B, Verma R, Konrad K. Altered brain network integrity after childhood maltreatment: A structural connectomic DTI-study. Hum Brain Mapp 2017;38:855-68.

23. McLaughlin KA, Weissman D, Bitrán D. Childhood Adversity and Neural Development: A Systematic Review. Annu Rev Dev Psychol 2019;1:277-312.

24. McLaughlin KA, Sheridan MA. Beyond Cumulative Risk: A Dimensional Approach to Childhood Adversity. Curr Dir Psychol Sci 2016;25:239-45.

25. Dennison MJ, Rosen ML, Sambrook KA, Jenness JL, Sheridan MA, McLaughlin KA. Differential Associations of Distinct Forms of Childhood Adversity With Neurobehavioral Measures of Reward Processing: A Developmental Pathway to Depression. Child Dev 2019;90:e96-e113.

26. Cancel LM, Arias K, Bikson M, Tarbell JM. Direct current stimulation of endothelial monolayers induces a transient and reversible increase in transport due to the electroosmotic effect. Sci Rep 2018;8:9265.

27. Quidé Y, Bortolasci CC, Spolding B, Kidnapillai S, Watkeys OJ, Cohen-Woods S, Berk M, Carr VJ, Walder $\mathrm{K}$, Green MJ. Association between childhood trauma exposure and pro-inflammatory cytokines in schizophrenia and bipolar-I disorder. Psychol Med 2019;49:2736-44.

28. Pollak SD, Cicchetti D, Hornung K, Reed A. Recognizing emotion in faces: developmental effects of child abuse and neglect. Dev Psychol 2000;36:679-88.

29. Sheridan MA, Peverill M, Finn AS, McLaughlin KA. Dimensions of childhood adversity have distinct associations with neural systems underlying executive functioning. Dev Psychopathol 2017;29:1777-94.

30. Blair KS, Aloi J, Crum K, Meffert H, White SF, Taylor BK, Leiker EK, Thornton LC, Tyler PM, Shah N, Johnson K, Abdel-Rahim H, Lukoff J, Dobbertin M, Pope K, Pollak S, Blair RJ. Association of Different Types of Childhood Maltreatment With Emotional Responding and Response Control Among Youths. JAMA Netw Open 2019;2:e194604.

31. Cassiers LLM, Sabbe BGC, Schmaal L, Veltman DJ, Penninx BWJH, Van Den Eede F. Structural and Functional Brain Abnormalities Associated With Exposure to Different Childhood Trauma Subtypes: A Systematic Review of Neuroimaging Findings. Front Psychiatry 2018;9:329.

32. Lambert HK, King KM, Monahan KC, McLaughlin KA. Differential associations of threat and deprivation with emotion regulation and cognitive control in adolescence. Dev Psychopathol 2017;29:929-40.

33. McLaughlin KA, Sheridan MA, Lambert HK. Childhood adversity and neural development: deprivation and threat as distinct dimensions of early experience. Neurosci Biobehav Rev 2014;47:578-91.

34. Busso DS, McLaughlin KA, Sheridan MA. Dimensions of Adversity, Physiological Reactivity, and Externalizing Psychopathology in Adolescence: Deprivation and Threat. Psychosom Med 2017;79:162-71.

35. Dube SR, Felitti VJ, Dong M, Giles WH, Anda RF. The impact of adverse childhood experiences on health problems: evidence from four birth cohorts dating back to 
1900. Prev Med 2003;37:268-77.

36. Nicol K, Pope M, Romaniuk L, Hall J. Childhood trauma, midbrain activation and psychotic symptoms in borderline personality disorder. Transl Psychiatry 2015;5:e559.

37. Busso DS, McLaughlin KA, Brueck S, Peverill M, Gold AL, Sheridan MA. Child Abuse, Neural Structure, and Adolescent Psychopathology: A Longitudinal Study. J Am Acad Child Adolesc Psychiatry 2017;56:321-328.e1.

38. Huang D, Liu Z, Cao H, Yang J, Wu Z, Long Y. Childhood trauma is linked to decreased temporal stability of functional brain networks in young adults. J Affect Disord 2021;290:23-30.

39. Bounoua N, Miglin R, Spielberg JM, Johnson CL, Sadeh N. Childhood trauma moderates morphometric associations between orbitofrontal cortex and amygdala: implications for pathological personality traits. Psychol Med 2020. [Epub ahead of print]. doi: 10.1017/ S0033291720004468.

40. Duncan NW, Hayes DJ, Wiebking C, Tiret B, Pietruska K, Chen DQ, Rainville P, Marjańska M, Ayad O, Doyon J, Hodaie M, Northoff G. Negative childhood experiences alter a prefrontal-insular-motor cortical network in healthy adults: A preliminary multimodal rsfMRI-fMRI-MRSdMRI study. Hum Brain Mapp 2015;36:4622-37.

41. Zhao W, Zhu D, Zhang Y, Zhang C, Zhang B, Yang Y, Zhu J, Yu Y. Relationship between illness duration, corpus callosum changes, and sustained attention dysfunction in major depressive disorder. Quant Imaging Med Surg 2021;11:2980-93.

42. Pijnenburg R, Scholtens LH, Mantini D, Vanduffel W, Barrett LF, van den Heuvel MP. Biological Characteristics of Connection-Wise Resting-State Functional Connectivity Strength. Cereb Cortex 2019;29:4646-53.

43. Tomasi D, Volkow ND. Functional connectivity density mapping. Proc Natl Acad Sci U S A 2010;107:9885-90.

44. Yeo BT, Krienen FM, Eickhoff SB, Yaakub SN, Fox PT, Buckner RL, Asplund CL, Chee MW. Functional Specialization and Flexibility in Human Association Cortex. Cereb Cortex 2016;26:465.

45. Saleh A, Potter GG, McQuoid DR, Boyd B, Turner R, MacFall JR, Taylor WD. Effects of early life stress on depression, cognitive performance and brain morphology. Psychol Med 2017;47:171-81.

46. Tian T, Li J, Zhang G, Wang J, Liu D, Wan C, Fang J, Wu D, Zhou Y, Zhu W. Effects of childhood trauma experience and BDNF Val66Met polymorphism on brain plasticity relate to emotion regulation. Behav Brain Res 2021;398:112949.
47. Bernstein DP, Ahluvalia T, Pogge D, Handelsman L. Validity of the Childhood Trauma Questionnaire in an adolescent psychiatric population. J Am Acad Child Adolesc Psychiatry 1997;36:340-8.

48. Everaerd D, Klumpers F, Zwiers M, Guadalupe T, Franke B, van Oostrom I, Schene A, Fernández G, Tendolkar I. Childhood abuse and deprivation are associated with distinct sex-dependent differences in brain morphology. Neuropsychopharmacology 2016;41:1716-23.

49. Tomasi D, Volkow ND. Association between functional connectivity hubs and brain networks. Cereb Cortex 2011;21:2003-13.

50. Chen SY, Cai GQ, Liang RB, Yang QC, Min YL, Ge QM, Li B, Shi WQ, Li QY, Zeng XJ, Shao Y. Regional brain changes in patients with diabetic optic neuropathy: a resting-state functional magnetic resonance imaging study. Quant Imaging Med Surg 2021;11:2125-37.

51. Tian T, Guo L, Xu J, Zhang S, Shi J, Liu C, Qin Y, Zhu W. Brain white matter plasticity and functional reorganization underlying the central pathogenesis of trigeminal neuralgia. Sci Rep 2016;6:36030.

52. Whitfield-Gabrieli S, Nieto-Castanon A. Conn: a functional connectivity toolbox for correlated and anticorrelated brain networks. Brain Connect 2012;2:125-41.

53. Klein A, Tourville J. 101 labeled brain images and a consistent human cortical labeling protocol. Front Neurosci 2012;6:171.

54. Barnes J, Ridgway GR, Bartlett J, Henley SM, Lehmann M, Hobbs N, Clarkson MJ, MacManus DG, Ourselin S, Fox NC. Head size, age and gender adjustment in MRI studies: a necessary nuisance? Neuroimage 2010;53:1244-55.

55. Milad MR, Quinn BT, Pitman RK, Orr SP, Fischl B, Rauch SL. Thickness of ventromedial prefrontal cortex in humans is correlated with extinction memory. Proc Natl Acad Sci U S A 2005;102:10706-11.

56. Roberts G, Green MJ, Breakspear M, McCormack C, Frankland A, Wright A, Levy F, Lenroot R, Chan HN, Mitchell PB. Reduced inferior frontal gyrus activation during response inhibition to emotional stimuli in youth at high risk of bipolar disorder. Biol Psychiatry 2013;74:55-61.

57. Bloom JS, Garcia-Barrera MA, Miller CJ, Miller SR, Hynd GW. Planum temporale morphology in children with developmental dyslexia. Neuropsychologia 2013;51:1684-92.

58. Cheng W, Rolls ET, Gu H, Zhang J, Feng J. Autism: reduced connectivity between cortical areas involved in 
face expression, theory of mind, and the sense of self. Brain 2015;138:1382-93.

59. Ho TC, Connolly CG, Henje Blom E, LeWinn KZ, Strigo IA, Paulus MP, Frank G, Max JE, Wu J, Chan M, Tapert SF, Simmons AN, Yang TT. Emotion-Dependent Functional Connectivity of the Default Mode Network in Adolescent Depression. Biol Psychiatry 2015;78:635-46.

60. Cao L, Xu J, Yang X, Li X, Liu B. Abstract Representations of Emotions Perceived From the Face, Body, and WholePerson Expressions in the Left Postcentral Gyrus. Front Hum Neurosci 2018;12:419.

61. Peyron R, Laurent B, García-Larrea L. Functional imaging of brain responses to pain. A review and metaanalysis (2000). Neurophysiol Clin 2000;30:263-88.

62. Takehara-Nishiuchi K. Entorhinal cortex and consolidated memory. Neurosci Res 2014;84:27-33.

63. Bonini F, Burle B, Liégeois-Chauvel C, Régis J, Chauvel P, Vidal F. Action monitoring and medial frontal cortex: leading role of supplementary motor area. Science 2014;343:888-91.

64. Liu H, Chen L, Zhang G, Jiang Y, Qu S, Liu S, Huang Y, Chen J. Scalp Acupuncture Enhances the Functional Connectivity of Visual and Cognitive-Motor Function Network of Patients with Acute Ischemic Stroke. Evid Based Complement Alternat Med 2020;2020:8836794.

65. Trick L, Kempton MJ, Williams SC, Duka T. Impaired fear recognition and attentional set-shifting is associated with brain structural changes in alcoholic patients. Addict Biol 2014;19:1041-54.

66. Lim L, Radua J, Rubia K. Gray matter abnormalities in childhood maltreatment: a voxel-wise meta-analysis. Am J Psychiatry 2014;171:854-63.

67. Mavridis I. The role of the nucleus accumbens in psychiatric disorders. Psychiatriki 2015;25:282-94.

68. Ma K, Zhang H, Wang S, Wang H, Wang Y, Liu J, Song
X, Dong Z, Han X, Zhang Y, Li H, Rahaman A, Wang $\mathrm{S}$, Baloch $Z$. The molecular mechanism underlying GABAergic dysfunction in nucleus accumbens of depression-like behaviours in mice. J Cell Mol Med 2019;23:7021-8.

69. Ou Y, Su Q, Liu F, Ding Y, Chen J, Zhang Z, Zhao J, Guo W. Increased Nucleus Accumbens Connectivity in Resting-State Patients With Drug-Naive, First-Episode Somatization Disorder. Front Psychiatry 2019;10:585.

70. Guo Z, Chen J, Liu S, Li Y, Sun B, Gao Z. Brain areas activated by uncertain reward-based decision-making in healthy volunteers. Neural Regen Res 2013;8:3344-52.

71. Kim EJ, Son JW, Park SK, Chung S, Ghim HR, Lee S, Lee SI, Shin CJ, Kim S, Ju G, Park H, Lee J. Cognitive and Emotional Empathy in Young Adolescents: an fMRI Study. Soa Chongsonyon Chongsin Uihak 2020;31:121-30.

72. Diana RA. Parahippocampal Cortex Processes the Nonspatial Context of an Event. Cereb Cortex 2017;27:1808-16.

73. Chirles TJ, Reiter K, Weiss LR, Alfini AJ, Nielson KA, Smith JC. Exercise Training and Functional Connectivity Changes in Mild Cognitive Impairment and Healthy Elders. J Alzheimers Dis 2017;57:845-56.

74. Buckner RL, Andrews-Hanna JR, Schacter DL. The brain's default network: anatomy, function, and relevance to disease. Ann N Y Acad Sci 2008;1124:1-38.

75. He X, Li X, Fu J, Xu J, Liu H, Zhang P, Li W, Yu C, Ye Z, Qin W. The morphometry of left cuneus mediating the genetic regulation on working memory. Hum Brain Mapp 2021;42:3470-80.

76. Grassi-Oliveira R, de Azevedo Gomes CF, Stein LM. False recognition in women with a history of childhood emotional neglect and diagnose of recurrent major depression. Conscious Cogn 2011;20:1127-34.
Cite this article as: Li J, Zhang G, Wang J, Liu D, Wan C, Fang J, Wu D, Zhou Y, Tian T, Zhu W. Experience-dependent associations between distinct subtypes of childhood trauma and brain function and architecture. Quant Imaging Med Surg 2022;12(2):1172-1185. doi: 10.21037/qims-21-435 